\title{
Three Dimensional Characterization of Cells in Hydrogel with Focused Ion Beam
}

\author{
A. Al-Abboodi ${ }^{1}$, J. Fu ${ }^{2}$, P.P.Y. Chan ${ }^{1}$ \& P. M. Doran ${ }^{1,3}$ \\ ${ }^{1}$ Department of Chemical Engineering, Monash University, Clayton, Australia, 3800 \\ 2 Department of Mechanical and Aerospace Engineering, Monash University, Clayton, \\ Australia, 3800 \\ ${ }^{3}$ School of Biological Sciences, Monash University, Clayton, Australia, 3800
}

Hydrogels are synthetic or natural polymer networks that have emerged as promising candidates for engineered complex 3D tissue scaffolds with tissue-like stiffness and biocompatibility [1]. With high permeability for oxygen, nutrients and other watersoluble metabolites through their high water-content matrix, hydrogels provide an excellent environment for cell growth and tissue regeneration because of their similarities to native extracellular matrix. In the past several years, research interest has shifted from hydrogel implants to injectable formulations which are advantageous [2], since cells and bioactive compounds, such as drugs, may be easily mixed with the precursor solutions prior to gelation to give homogeneously loaded gels. Moreover, in situ gelation allows for minimally invasive surgery with the possibility of complex shapes.

To image the cell growth in situ is crucial to understand and validate the proposed hydrogel designs, but there are still a few hurdles to clear. The porous microstructures, with or without residing cells, are not suitable for microtomy, and thus high resolution imaging is still limited to study the cell-hydrogel interfaces. Moreover, 3D investigation on cells/hydrogel simultaneously is still difficult with existing imaging techniques, such as confocal microscopy, due to lacking of co-staining methods. Initial attempts on imaging cell-material interface by FIB milling cross sections have been presented [3] [4], although actual 3D information is not obtained.

In our study, hydrogel precursor solution was mixed with African green monkey kidney cells (COS-7 cells) followed by polymerization by adding Horseradish peroxidase (HRP) and diluted $\mathrm{H}_{2} \mathrm{O}_{2}$. For vacuum based analysis, the samples were fixed with plunge freeze and then transferred to freeze drying. Additional aliquots for high resolution imaging were stained with Osmium tetroxide. We demonstrate that using focused ion beam milling and associated microanalysis techniques including SEM, EDX, etc., three dimensional characterizations can be effectively performed on cells grown inside hydrogel. In addition, 3D reconstruction with FIB/SEM tomography [5] can provide accurate statistics of the porous microstructures, and their implications for cell proliferation as well.

\section{References}

[1] J. da Silva et al., Biomaterials, 31 (2010) 2201

[2] J. D. Kretlow et al., Adv. Drug Deliv. Rev., 59 (2007) 263 
[3] A. G. Bittermann, et al., Adv. Eng. Mater., 11 (2009) 182

[4] E. Martínez, et al., Micron, 39 (2008) 111

[5] J.A. Heymann et al., J. Struct. Biol., 155 (2006) 63
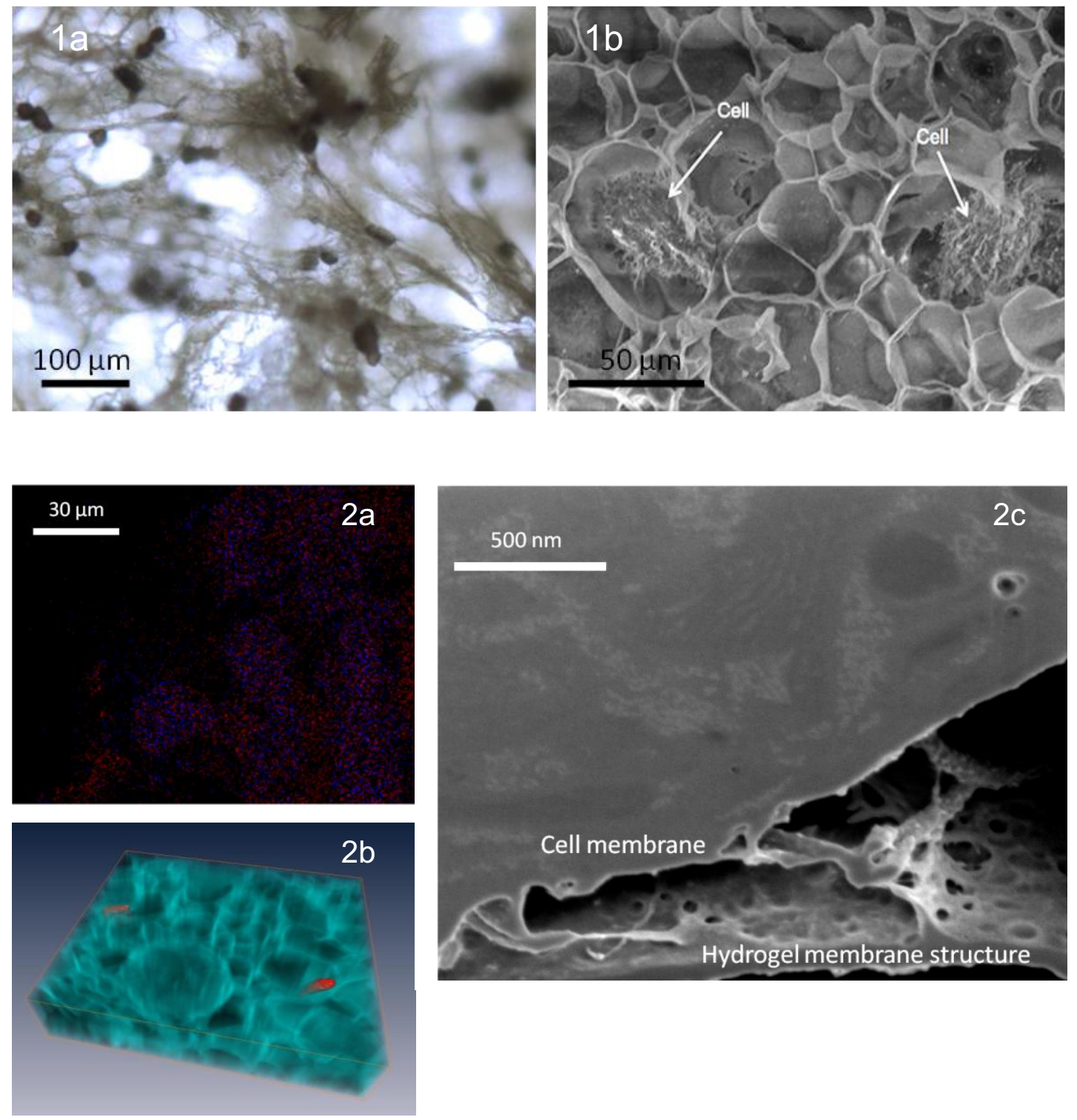

Hydrogel membrane structure

FIG. 1. (a) Photomicrograph of African green monkey kidney cells (COS-7 cells) in hydrogel and (b) corresponding SEM images after freeze drying.

FIG. 2. Imaging cells in hydrogel with FIB milling (a) SEM-EDX image of the exposed cells (Overlay of $\mathrm{Na}, \mathrm{O}$ and $\mathrm{P}$ ) (b) Rendering of a volume $(84 \times 70 \times 17 \mu \mathrm{m}$ from 137 images) based on FIB/SEM tomography showing cells (red) grown in hydrogel microstructures (cyan). (c) High magnification SEM image on cell-hydrogel interfaces. 